\title{
Single- and multiple-dose pharmacokinetics, pharmacodynamics, and safety of apixaban in healthy Chinese subjects
}

This article was published in the following Dove Press journal:

Clinical Pharmacology:Advances and Applications

4 December 2013

Number of times this article has been viewed

\author{
Yimin Cui' \\ Yan Song ${ }^{2}$ \\ Jessie Wang ${ }^{2}$ \\ Zhigang $\mathrm{Yu}^{2}$ \\ Alan Schuster ${ }^{2}$ \\ Yu Chen Barrett ${ }^{2}$ \\ Charles Frost ${ }^{2}$
}

'Peking University First Hospital, Beijing, People's Republic of China; ${ }^{2}$ Bristol-Myers Squibb, Princeton, $\mathrm{NJ}$, USA
Correspondence: Charles Frost Bristol-Myers Squibb (EI2-16), Route 206 and Province Line Road, Princeton, NJ 08543, USA

Tel + I 6092523552

Fax +l 6092526313

Email charles.frost@bms.com
Background: The pharmacokinetics (PK), pharmacodynamics (PD), and safety of apixaban were assessed in healthy Chinese subjects in this randomized, placebo-controlled, double-blind, single-sequence, single- and multiple-dose study.

Subjects and methods: Eighteen subjects 18-45 years of age were randomly assigned (2:1 ratio) to receive apixaban or matched placebo. Subjects received a single $10 \mathrm{mg}$ dose of apixaban or placebo on day 1 , followed by $10 \mathrm{mg}$ apixaban or placebo twice daily for 6 days (days 4-9). The PK and PD of apixaban were assessed by collecting plasma samples for 72 hours following the dose on day 1 and the morning dose on day 9, and measuring apixaban concentration and anti-Xa activity. Safety was assessed via physical examinations, vital sign measurements, electrocardiograms, and clinical laboratory evaluations.

Results: PK analysis showed similar characteristics of apixaban after single and multiple doses, including a median time to maximum concentration of $\sim 3$ hours, mean elimination half-life of $\sim 11$ hours, and renal clearance of $\sim 1.2 \mathrm{~L} /$ hour. The accumulation index was 1.7 , consistent with twice-daily dosing and the observed elimination half-life. Single-dose data predict multipledose PK, therefore apixaban PK are time-independent. The relationship between anti-Xa activity and plasma apixaban concentrations appears to be linear. Apixaban was safe and well tolerated, with no bleeding-related adverse events reported.

Conclusion: Apixaban was safe and well tolerated in healthy Chinese subjects. Apixaban PK and PD were predictable and consistent with findings from previous studies in Asian and non-Asian subjects. The administration of apixaban does not require any dose modification based on race.

Keywords: apixaban, oral anticoagulant, factor $\mathrm{Xa}$ inhibitor, pharmacokinetics, pharmacodynamics

\section{Introduction}

Until recently, available anticoagulant treatments included vitamin $\mathrm{K}$ antagonists, such as warfarin, and heparinoids, such as unfractionated heparin and low-molecularweight heparin. Apixaban is a novel oral anticoagulant recently approved in several countries for thromboprophylaxis following elective knee- or hip-replacement surgery and for prevention of stroke or systemic embolism in patients with nonvalvular atrial fibrillation. ${ }^{1-5}$ Apixaban is also being developed for the treatment of venous thromboembolism. ${ }^{6,7}$

Variations in the pharmacokinetic and pharmacodynamic properties of anticoagulants such as heparin and warfarin, as well as resulting clotting times, were observed among different racial groups, including different Asian ethnicities. ${ }^{8-10}$ For example, 
genetic polymorphism of cytochrome P450 (CYP) 2C9 as well as vitamin $\mathrm{K}$ epoxide reductase are both responsible for the lower warfarin maintenance doses among the three major Asian ethnic groups (Chinese, Malay, and Indian). ${ }^{9,10}$ In addition, certain characteristics, such as metabolism by enzymes known to show genetic polymorphism, high intersubject variation in bioavailability, or low bioavailability, may make a drug more likely to be affected by ethnic factors. ${ }^{11}$ Since there may be concerns regarding variations in the pharmacokinetics (PK) and pharmacodynamics (PD) of anticoagulants among different Asian ethnic groups, the PK, PD, and safety of apixaban were evaluated in healthy Chinese subjects. The primary objective of this study was to assess the PK of single- and multiple-dose apixaban. Secondary objectives included evaluating both safety and the effects of apixaban on PD markers of coagulation.

\section{Subjects and methods}

Healthy male and female Chinese subjects 18-45 years of age with a body mass index of $19-24 \mathrm{~kg} / \mathrm{m}^{2}$ were enrolled. Exclusion criteria included any significant or acute medical illness or relevant trauma, gastrointestinal disease, or surgery in the previous 3 months that could impact drug absorption; a history of abnormal bleeding or coagulation disorder; any significant head injury within the previous 2 years; any major surgery within the 4 weeks prior to dosing or planned within 2 weeks after completion of the study; or any history of acute or chronic pancreatitis. Female subjects were excluded if pregnant or breastfeeding, and were required to have a negative serum or urine pregnancy test within 24 hours prior to dosing. All subjects were required to use an acceptable method of contraception for the duration of the study.
Subjects were also excluded for a recent history of smoking or alcohol abuse.

The study was conducted in accordance with Good Clinical Practice (as defined by the International Conference on Harmonisation) and with the ethical principles of the Declaration of Helsinki, EU Directive 2001/20/EC, the US Code of Federal Regulations, Title 21, Part 50 (21CFR50), and all applicable local regulatory requirements. The protocol and subject informed-consent forms received appropriate approval by the Institutional Review Board/Independent Ethics Committee of Peking University First Hospital (Beijing, People's Republic of China) before the study began. Written informed consent was obtained from all subjects prior to screening.

\section{Study design}

This was a randomized, placebo-controlled, double-blind, single-sequence, single- and multiple-dose study in healthy Chinese subjects (Figure 1). All subjects were screened and evaluated for eligibility within 21 days prior to study drug administration. Enrolled subjects who met all eligibility criteria at screening were randomly assigned in a 2:1 ratio to receive either apixaban or matched placebo. Randomization was carried out according to a computer-generated randomization scheme supplied by the sponsor.

Subjects were admitted to the clinical facility the day before study-drug administration and were confined to the facility for the duration of the study. Each subject received either a single $10 \mathrm{mg}$ oral dose of apixaban $(2 \times 5 \mathrm{mg}$ tablets) or matched placebo on day 1 of the study. From days 4 to 8 , each subject received either oral apixaban $10 \mathrm{mg}$ twice daily or matched placebo, given at approximately

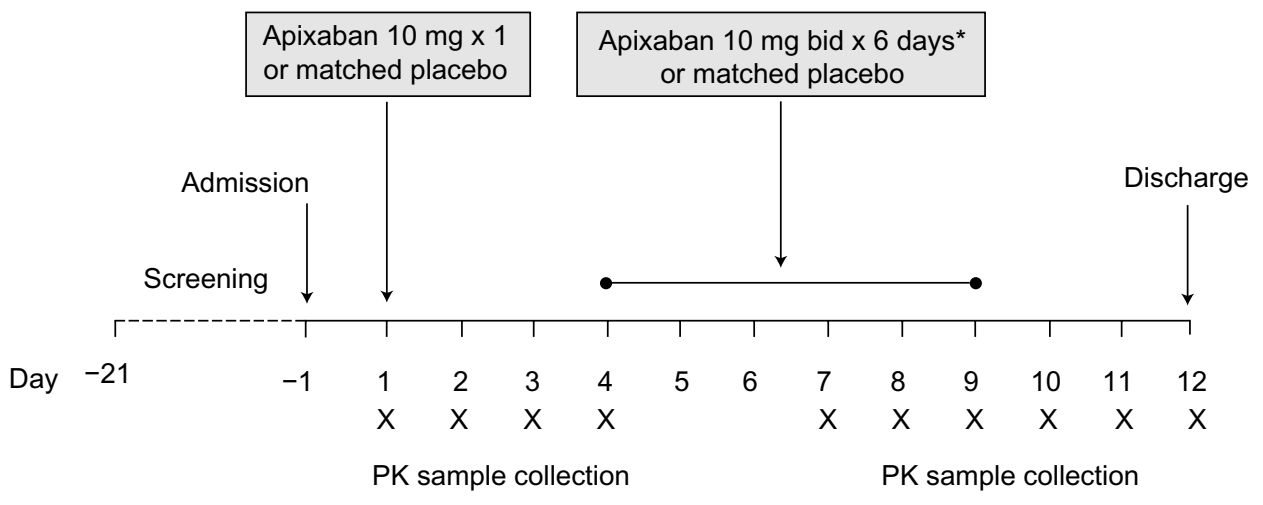

Figure I Study design.

Note: *Ending with the morning dose on day 9.

Abbreviations: bid, twice daily; PK, pharmacokinetic. 
9 am and $9 \mathrm{pm}$ in the fasted state. The last dose of study drug was administered on the morning of day 9. Subjects were discharged from the study after completion of clinical and laboratory safety evaluations on day 12 . Blood and urine samples were collected over the course of the study for clinical laboratory evaluations. Standard meals were served during the study.

\section{Sample collection and analysis}

Blood samples for PK and PD assessments were collected using an indwelling catheter or direct venipuncture predose on days $1,7,8$, and 9 , and $0.5,1,2,3,4,6,9,12,24,36,48$, 60 , and 72 hours following the morning dose of apixaban on days 1 and 9. For PK analysis, each $2.7 \mathrm{~mL}$ blood sample was completely mixed immediately after collection with a $3.2 \%$ sodium citrate anticoagulant and then placed on chipped ice. Within 15 minutes, each blood sample was centrifuged for 15 minutes at approximately $1,500 \times g$ at $4^{\circ} \mathrm{C}$ to separate plasma. Separated plasma was transferred to cryogenic vials, immediately stored at or below $-20^{\circ} \mathrm{C}$, and sent for analysis to Intertek Pharmaceutical Services (formerly Alta Analytical Laboratory), El Dorado, CA, USA. No PK or PD samples from subjects receiving matched placebo were analyzed.

Urine was collected on days 1-4 (predose, 0-12, 12-24, 24-48, and 48-72 hours relative to the day 1 morning dose) and on day 9 ( $0-12$ hours relative to the morning dose) for the analysis of apixaban. Urine was collected in chilled collection jugs and stored under refrigerated conditions. Aliquots $(10 \mathrm{~mL})$ of each sample were transferred into polypropylene vials and stored at or below $-20^{\circ} \mathrm{C}$. The samples were sent to Intertek Pharmaceutical Services for analysis.

Apixaban concentration was measured in plasma and urine samples using a validated high-performance liquid chromatography atmospheric pressure ionization tandem mass spectrometry method. Sample extraction for plasma utilized protein precipitation, and for urine, solid-phase extraction was employed. Apixaban-M4, a stable-label internal standard, was used for both assays. The lower limit of quantification was $1.0 \mathrm{ng} / \mathrm{mL}$ for both apixaban plasma and urine assays. The between-run and within-run variabilities for apixaban in plasma quality-control samples, expressed as coefficient of variation (CV), were $\leq 5.97 \%$ and $\leq 7.84 \%$, respectively, with deviations from nominal concentration of no more than $\pm 2.15 \%$. The between-run and within-run variabilities for apixaban in urine quality-control samples were $\leq 13.5 \%$ and $\leq 20.1 \%$, respectively, with deviations from nominal concentration of no more than $\pm 7.56 \%$. All samples were analyzed within the period of analyte stability.
For PD analysis, blood samples were collected in $4.5 \mathrm{~mL}$ $3.2 \%$ sodium citrate tubes and immediately gently mixed by inversion before being placed on ice. Within 30 minutes of blood collection, samples were centrifuged either for 15 minutes at $2,500 \times g$ or 20 minutes at $1,500 \times g$ at $4^{\circ} \mathrm{C}$ to separate plasma. Separated plasma samples were stored in vials at $-70^{\circ} \mathrm{C}$ until analyzed. Anti-Xa activity was measured by a validated chromogenic assay at Esoterix Coagulation Laboratory (Aurora, CO, USA). ${ }^{12}$ The one-step competition reaction involved a fixed amount of bovine factor Xa, added to a mixture of citrated human plasma and synthetic para-nitroaniline (pNA)-containing peptide substrate with exogenous antithrombin added. The quantity of pNA released was inversely proportional to the concentration of factor Xa inhibitor present in the sample mixture. The results of the assay were reported in low-molecular-weight heparin units (range $0.2-18.4 \mathrm{IU} / \mathrm{mL}$ ).

\section{Pharmacokinetic and pharmacodynamic analysis}

Individual subject PK parameters were derived by noncompartmental methods (Kinetica, version 4.4.1 within the eToolbox EP, version 2.6.1; Thermo Fisher Scientific, Waltham, MA, USA). Single- and multiple-dose PK parameters assessed included maximum observed plasma concentration $\left(\mathrm{C}_{\text {max }}\right)$ and time to maximum plasma concentration $\left(\mathrm{T}_{\max }\right)$. The observed plasma concentrations at trough $\left(\mathrm{C}_{\min }\right)$ at 12 hours postdose on day 9 were also recorded. Plasma half-life $\left(t_{1 / 2}\right)$ was estimated as $\ln 2 / \lambda$, where $\lambda$ was the terminal phase of the plasma concentration-time profile determined with a weighting factor of 1 using the method of least squares (log-linear regression of at least 3 data points). Area under the plasma concentration-time curve (AUC) from time 0 to time of the last quantifiable concentration following a single dose $\left(\mathrm{AUC}_{0-t}\right)$ was calculated using conventional trapezoidal and log-trapezoidal methods. AUC from time 0 extrapolated to infinite time following a single dose $\left(\mathrm{AUC}_{\infty}\right)$ was determined by summing $\mathrm{AUC}_{0-t}$, and the extrapolated area determined by dividing the last measured concentration by the slope of the terminal log-linear phase. The AUC in one dosing interval $\left(\mathrm{AUC}_{\tau}\right)$ was determined by summing the areas from time 0 to the time of next dose (0-12 hours), using conventional trapezoidal and log-trapezoidal methods. The accumulation index (AI) was calculated as the ratio of $\mathrm{AUC}_{\tau}$ on day 9 divided by $\mathrm{AUC}_{\tau}$ on day 1 . Renal clearance $\left(\mathrm{CL}_{\mathrm{R}}\right)$ was calculated as urinary recovery $\left(\mathrm{UR}_{t}\right) / \mathrm{AUC}_{0-t}$, with $\mathrm{UR}_{t}$ being the amount of drug collected in the same time interval (time $0-t$ ). 
Mean plasma anti-Xa activity profiles were derived from individual data obtained on day 9 over one dosing interval (12 hours). Maximum and minimum anti-Xa activities for apixaban were determined from the mean plasma anti-Xa activity data.

\section{Safety}

Subjects were closely monitored for adverse events, and were not discharged from the study until the investigator had determined that all adverse events had resolved or were not of clinical significance. Data on adverse events were collected through constant monitoring, volunteering of information by the study participants, and daily questioning by the medical staff. Additionally, adverse events could be identified by investigator review of vital signs, electrocardiogram (ECG), laboratory, and other data.

\section{Statistics}

The number of subjects was not based on statistical power considerations. However, administration of apixaban to twelve subjects would provide $90 \%$ confidence that the estimated apixaban $\mathrm{C}_{\max }$ and $\mathrm{AUC}$ geometric means would be within $17 \%$ and $16 \%$, respectively, of true population values. These calculations assumed that $\mathrm{C}_{\max }$ and $\mathrm{AUC}_{0-t}$ were log-normally distributed with intersubject standard deviations (SDs) of 0.33 and 0.31 for $\ln \left(\mathrm{C}_{\max }\right)$ and $\ln \left(\mathrm{AUC}_{0-t}\right)$, respectively. ${ }^{13}$ Summary statistics for each PK parameter were tabulated by study day, with geometric means and $\mathrm{CV}$ presented for both single- and multiple-dose PK parameters (including $\mathrm{C}_{\max }, \mathrm{AUC}_{0-t}, \mathrm{AUC}_{\infty}, \mathrm{AUC}_{\tau}$, and AI). Minima, medians, and maxima were presented for $\mathrm{T}_{\max }$, and means and SDs determined for $t_{1 / 2}$ and $\mathrm{CL}_{\mathrm{R}}$. The relationships between apixaban concentration and anti-Xa activity after single and multiple doses of apixaban were explored. A scatter plot of plasma concentration versus anti-Xa activity was plotted with a fitted linear regression line. All adverse events recorded during the study were listed and tabulated by the Medical Dictionary for Regulatory Activities (version 11), system organ class, preferred term, and treatment.

\section{Results Disposition}

Eighteen healthy Chinese subjects (twelve men and six women) between the ages of 28 and 39 years were enrolled. All subjects completed the study. A summary of subject demographics is shown in Table 1.
Table I Baseline demographics

\begin{tabular}{|c|c|c|}
\hline & Apixaban $(n=\mid 2)$ & Placebo $(n=6)$ \\
\hline \multicolumn{3}{|l|}{ Sex, n (\%) } \\
\hline Male & $8(67)$ & $4(67)$ \\
\hline Female & $4(33)$ & $2(33)$ \\
\hline \multicolumn{3}{|l|}{ Age, years } \\
\hline Mean (SD) & $35(3)$ & $32(4)$ \\
\hline Range & $32-39$ & $28-38$ \\
\hline \multicolumn{3}{|l|}{ Race, n (\%) } \\
\hline Asian & $12(100)$ & $6(100)$ \\
\hline \multicolumn{3}{|c|}{ Body weight, kg } \\
\hline Mean (SD) & $63.1(5.0)$ & $61.4(7.3)$ \\
\hline Range & $54.0-68.0$ & $54.0-72.0$ \\
\hline \multicolumn{3}{|l|}{ Height, cm } \\
\hline Mean (SD) & $163.7(5.3)$ & $166.2(6.0)$ \\
\hline Range & $152.0-169.0$ & $156.0-174.5$ \\
\hline \multicolumn{3}{|l|}{$\mathrm{BMI}, \mathrm{kg} / \mathrm{m}^{2}$} \\
\hline Mean (SD) & $23.5(0.7)$ & $22.2(1.6)$ \\
\hline Range & $21.4-24.0$ & $19.5-23.6$ \\
\hline
\end{tabular}

Abbreviations: BMI, body mass index; SD, standard deviation.

\section{Pharmacokinetics}

Summary statistics for PK parameters for single- and multiple-dose apixaban are shown in Table 2. Apixaban was rapidly absorbed, with peak concentrations observed 3 hours (range 2-4 hours) after administration. The descending phase of the plasma concentration-time curves appeared to be biphasic, characterized by a relatively rapid initial descending phase followed by a slower terminal phase (Figure 2). The mean terminal $t_{1 / 2}$ was consistent following single- and multiple-dose administration (11.4 hours and 10.8 hours, respectively). The $\mathrm{CL}_{\mathrm{R}}$ was approximately $1.2 \mathrm{~L} /$ hour.

Following multiple doses of apixaban, predose trough concentrations did not change appreciably beyond day 7 (mean 111-145 ng/mL; mean day 9 predose and 12-hour postdose concentrations were $111 \mathrm{ng} / \mathrm{mL}$ and $121 \mathrm{ng} / \mathrm{mL}$, respectively), with an AI at steady state of approximately 1.7. The peak-to-trough ratio of mean concentration was approximately 2.9 .

\section{Pharmacodynamics}

The time course of anti-Xa activity was coincident with the apixaban concentration-time profile (Figure 3), with no evidence of temporal lag or difference between the single- and multiple-dose time courses observed. On day 9, maximum anti-Xa activity, corresponding to the peak apixaban concentration at 3 hours, was $5.57(\mathrm{SD}, 1.54) \mathrm{IU} / \mathrm{mL}$; minimum anti-Xa activity, coinciding with the trough apixaban concentration at 12 hours, was $1.94(\mathrm{SD}, 0.54) \mathrm{IU} / \mathrm{mL}$, based 


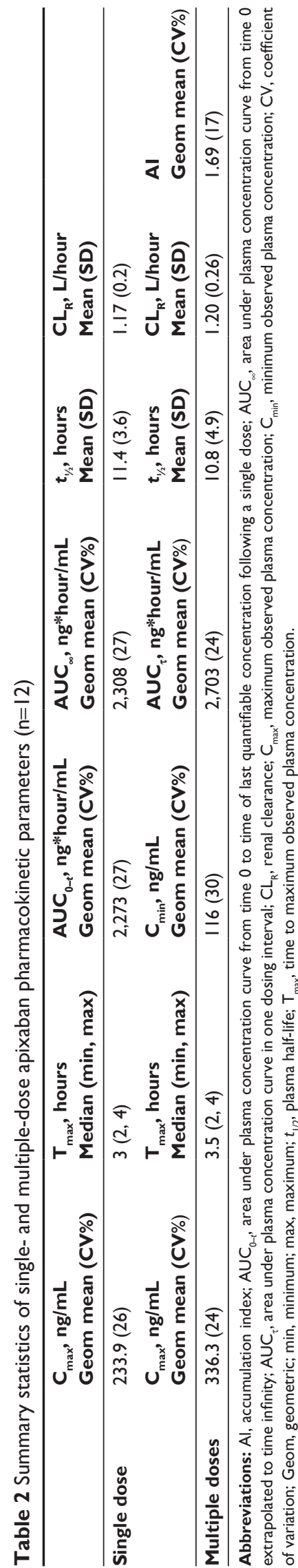

on mean measurement data. In addition, a direct linear relationship was observed between anti-Xa activity and plasma apixaban concentration (Figure 4).

\section{Safety}

There were no deaths, other serious adverse events, adverse events leading to discontinuation, or clinically significant bleeding-related adverse events reported during the study. No adverse events were reported following administration of single-dose apixaban $10 \mathrm{mg}$ or placebo. All adverse events in apixaban- and placebo-treated subjects following multipledose administration were mild, considered possibly related to treatment, and resolved without treatment. The types and frequencies of reported adverse events were generally similar between apixaban- and placebo-treated subjects. In total, three adverse events (elevated alanine aminotransferase [ALT], positive fecal blood, and microscopic hematuria) were reported in three subjects receiving apixaban (one of twelve subjects [8\%] for each), and two adverse events (elevated ALT and positive fecal blood) were reported in one subject receiving placebo (one of six subjects [17\%]). No clinically significant changes in vital signs, physical examinations, or ECGs were observed during the study. Results for activated partial thromboplastin time, prothrombin time, and international normalized ratio were within normal limits throughout the study.

\section{Discussion}

Apixaban undergoes rapid absorption after oral administration, attaining $\mathrm{C}_{\max } \sim 3$ hours after administration with an oral bioavailability of $\sim 50 \% .^{14,15}$ Apixaban has a mean elimination $t_{1 / 2}$ of approximately 12 hours in healthy Western subjects. ${ }^{14-16}$ Apixaban is cleared via multiple pathways, including metabolism, biliary, intestinal, and renal excretion; $27 \%$ of total clearance is via the kidneys and the remainder in feces. ${ }^{14,17,18}$ Following oral administration, most of the dose is excreted unchanged in the feces, although some undergoes oxidative metabolism ( $25 \%$ ), which is primarily mediated via CYP3A4 along with a minor contribution from other CYP enzymes. ${ }^{14,19,20}$ Apixaban is a substrate of P-glycoprotein and breast cancer resistance protein. ${ }^{21}$ Based on its PK and PD properties, apixaban has limited potential to be influenced by ethnicity. ${ }^{11}$ Therefore, the results of this study were expected to be consistent with the findings of similar studies conducted primarily in non-Asian subjects, ${ }^{22,23}$ and facilitate acceptance of safety and efficacy data from non-Asian subjects by health authorities in the Asia-Pacific regions. ${ }^{11}$ 

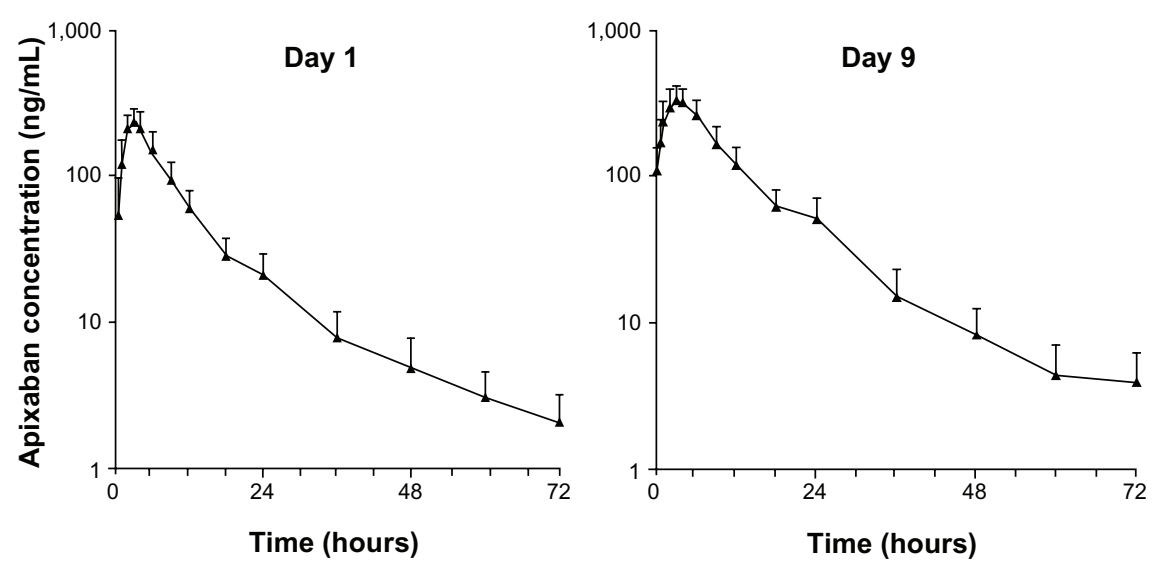

Figure 2 Mean plasma apixaban concentration versus time $(n=12)$ following single-dose administration (day I) and at steady state (day 9$)$. Error bars show $+\mathrm{I}$ standard deviation from the mean.
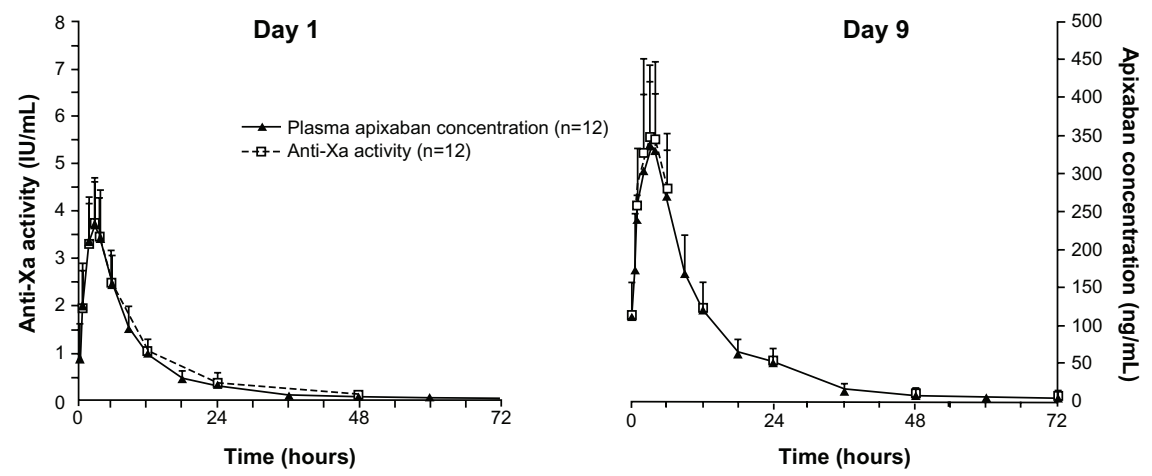

Figure 3 Mean anti-Xa activity and plasma apixaban concentration versus time following single-dose administration (day I) and at steady state (day 9). Error bars show + I standard deviation from the mean.

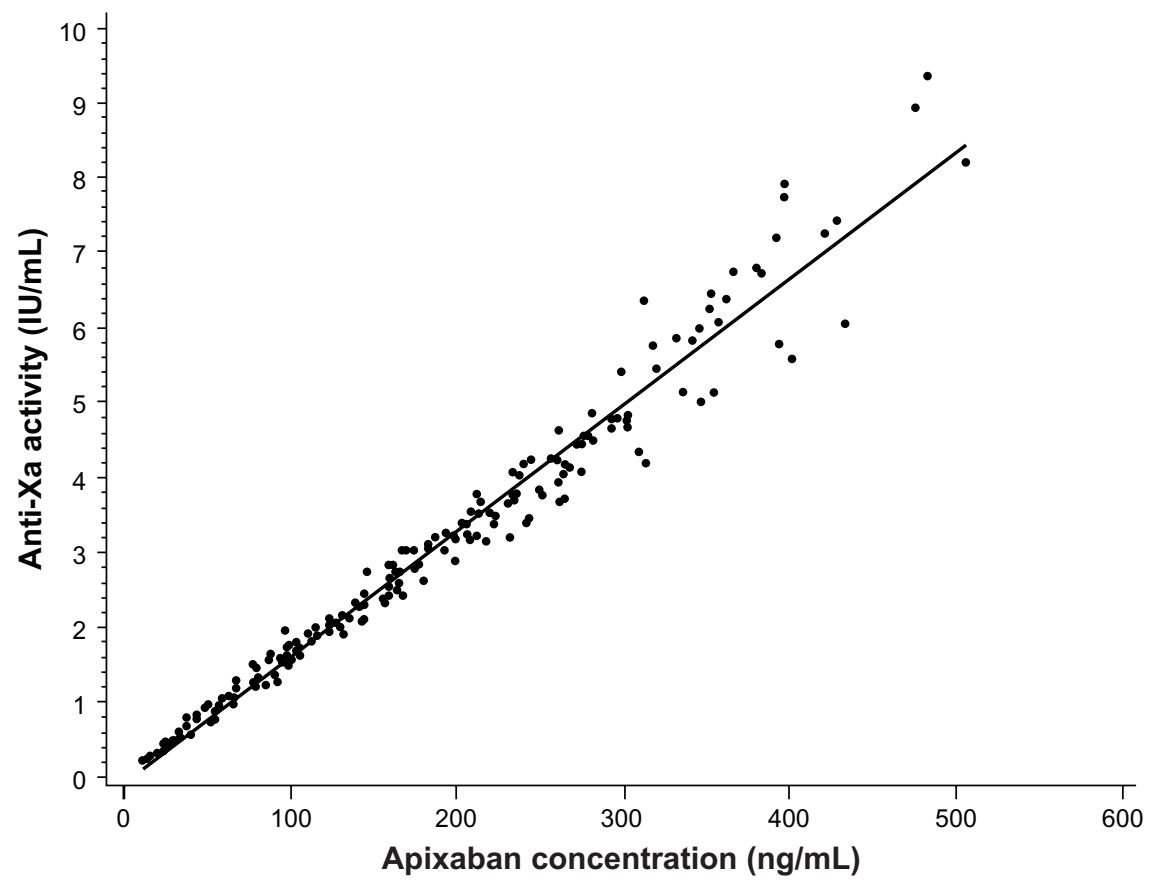

Figure 4 Scatter plot of anti-Xa activity versus plasma apixaban concentration. Anti-Xa activity values less than lower limit of quantitation are excluded. Note: Regression equation: Anti-Xa $=0.01690+0.09597 \times$ concentration. 
The results of this study confirm that apixaban PK and $\mathrm{PD}$ are consistent with findings in previous studies, including those conducted primarily in non-Asian subjects. Apixaban was well absorbed in Chinese subjects, with $\mathrm{C}_{\max }$ occurring 2-4 hours after administration. This rapid absorption is similar to that observed in white and Japanese subjects, in whom $\mathrm{C}_{\max }$ was reached in 2-4 hours..$^{15,22,23}$

The $t_{1 / 2}$ values for apixaban observed in Chinese subjects following single- and multiple-dose administration ( $\sim 11$ hours) were similar to those observed in white and Japanese subjects. ${ }^{15,16,22,23} \mathrm{CL}_{\mathrm{R}}$ was 1.17 and $1.20 \mathrm{~L}$ /hour following single and multiple doses, respectively, consistent with that observed in previous studies in Japanese and nonAsian subjects (0.49-1.07 L/hour). Apixaban accumulation after multiple doses was modest, $\mathrm{AI}=1.7$, consistent with the $\sim 11$-hour $t_{1 / 2}$ and frequency of administration and previous findings in healthy subjects. ${ }^{16}$ Intersubject variability in $\mathrm{AUC}$ and $\mathrm{C}_{\max }$ was low; the $\mathrm{CV}$ was $\leq 30 \%$ after single- and multiple-dose administration. AUCs following single- and multiple-dose administration were comparable to those observed in healthy subjects in the US and Japan. ${ }^{16,22,23}$ Trough concentrations did not change appreciably beyond day 7 , indicating steady-state conditions were achieved by the third day of multiple-dose administration. The peak-totrough ratio of 2.9 indicates minimal fluctuation in apixaban exposure with a twice-daily dosing regimen.

Administration of apixaban was associated with a fast onset of action and concentration-dependent increases in plasma anti-Xa activity, consistent with the expected effects of a direct factor Xa inhibitor. ${ }^{12,24}$ As observed in previous studies, the time course of anti-Xa activity closely tracked the apixaban plasma concentration-time profile, with a direct linear relationship between the two that exhibited no temporal lag. ${ }^{12,25}$ This is consistent with the parent compound being solely responsible for the observed anticoagulant activity, and is evident in the similarity between the anti-Xa activity peak-to-trough ratio (calculated based on observed maximum and minimum from the mean profile) of 2.9 and the apixaban concentration peak-to-trough ratio (2.9).

In summary, apixaban was safe and well tolerated in these healthy young Chinese subjects. The observed PK characteristics of apixaban were consistent with those reported for similar studies conducted in healthy Caucasian and Japanese subjects, and characterized by rapid absorption and a terminal $t_{1 / 2}$ of approximately 12 hours. Differences may exist between the PK or PD observed in this study versus what may be expected in the patient population, due to the influence of other intrinsic or extrinsic factors present in the patient population. How- ever, the potential influence of race is expected to be similar between patients and healthy young subjects. Therefore, the similarity between apixaban PK, PD, and tolerability observed in this study with that observed in previous healthy-subject studies conducted in the US and Japan indicates that no dose adjustment is needed based on race.

\section{Acknowledgments}

Professional editorial and medical writing support was provided by Andy Shepherd, PhD, and Dana Fox, PhD, CMPP, of Caudex Medical Ltd, and funded by Bristol-Myers Squibb Company and Pfizer Inc. The authors would like to thank Dr Tong Li for his support as medical monitor for the study.

\section{Disclosure}

This study was sponsored by Bristol-Myers Squibb Company and Pfizer Inc. YS, JW, ZY, AS, YCB, and CF had support from Bristol-Myers Squibb (employee/stock/stock options). YC is an employee of Peking University Hospital and declares no support from any organization for the submitted work.

\section{References}

1. Lassen MR, Raskob GE, Gallus A, Pineo G, Chen D, Portman RJ Apixaban or enoxaparin for thromboprophylaxis after knee replacement. N Engl J Med. 2009;361:594-604.

2. Lassen MR, Raskob GE, Gallus A, Pineo G, Chen D, Hornick P. Apixaban versus enoxaparin for thromboprophylaxis after knee replacement (ADVANCE-2): a randomised double-blind trial. Lancet. 2010;375:807-815.

3. Lassen MR, Gallus A, Raskob GE, Pineo G, Chen D, Ramirez LM. Apixaban versus enoxaparin for thromboprophylaxis after hip replacement. N Engl J Med. 2010;363:2487-2498.

4. Granger CB, Alexander JH, McMurray JJ, et al. Apixaban versus warfarin in patients with atrial fibrillation. $N$ Engl J Med. 2011;365: 981-992.

5. Connolly SJ, Eikelboom J, Joyner C, et al. Apixaban in patients with atrial fibrillation. $N$ Engl J Med. 2011;364:806-817.

6. Agnelli G, Buller HR, Cohen A, et al. Apixaban for extended treatment of venous thromboembolism. N Engl J Med. 2013;368:699-708.

7. Agnelli G, Buller HR, Cohen A, et al. Oral apixaban for the treatment of acute venous thromboembolism. N Engl J Med. 2013;369:799-808.

8. Shimada YJ, Nakra NC, Fox JT, Kanei Y. Relation of race (Asian, African-American, European-American, and Hispanic) to activated clotting time after weight-adjusted bolus of heparin during percutaneous coronary intervention. Am J Cardiol. 2010;105:629-632.

9. Yuen E, Gueorguieva I, Wise S, Soon D, Aarons L. Ethnic differences in the population pharmacokinetics and pharmacodynamics of warfarin. J Pharmacokinet Pharmacodyn. 2010;37:3-24.

10. Lee SC, $\mathrm{Ng} \mathrm{SS}$, Oldenburg J, et al. Interethnic variability of warfarin maintenance requirement is explained by VKORC1 genotype in an Asian population. Clin Pharmacol Ther. 2006;79:197-205.

11. International Conference on Harmonisation of Technical Requirements for Registration of Pharmaceuticals for Human Use. ICH Harmonised Tripartite Guideline: Ethnic factors in the acceptability of foreign clinical data - E5 (R1). 1998. Available from: http://www.ich.org/fileadmin/ Public_Web_Site/ICH_Products/Guidelines/Efficacy/E5_R1/Step4/ E5_R1_Guideline.pdf. Accessed October 3, 2013. 
12. Barrett YC, Wang Z, Frost C, Shenker A. Clinical laboratory measurement of direct factor Xa inhibitors: anti-Xa assay is preferable to prothrombin time assay. Thromb Haemost. 2010;104:1263-1271.

13. Frost C, Nepal S, Barrett Y, LaCreta F. Effects of age and gender on the single-dose pharmacokinetics (PK) and pharmacodynamics (PD) of apixaban. J Thromb Haemost. 2009;7:PP-MO-407.

14. Raghavan N, Frost CE, Yu Z, et al. Apixaban metabolism and pharmacokinetics after oral administration to humans. Drug Metab Dispos. 2009;37:74-81.

15. Frost C, Wang J, Nepal S, et al. Apixaban, an oral, direct factor Xa inhibitor: single dose safety, pharmacokinetics, pharmacodynamics and food effect in healthy subjects. Br J Clin Pharmacol. 2013;75: 476-487.

16. Frost C, Nepal S, Wang J, et al. Safety, pharmacokinetics and pharmacodynamics of multiple oral doses of apixaban, a factor Xa inhibitor, in healthy subjects. Br J Clin Pharmacol. Epub March 4, 2013.

17. Zhang D, Frost CE, He K, et al. Investigating the enteroenteric recirculation of apixaban, a factor Xa inhibitor: administration of activated charcoal to bile duct-cannulated rats and dogs receiving an intravenous dose and use of drug transporter knockout rats. Drug Metab Dispos. 2013;41:906-915.

18. Frost C, Yu Z, Nepal S, et al. Apixaban, a direct factor Xa inhibitor: single-dose pharmacokinetics and pharmacodynamics of an intravenous formulation [abstract 148]. J Clin Pharmacol. 2008;48:1132.
19. Wang L, Zhang D, Raghavan N, et al. In vitro assessment of metabolic drug-drug interaction potential of apixaban through cytochrome P450 phenotyping, inhibition, and induction studies. Drug Metab Dispos. 2010;38:448-458.

20. Zhang D, He K, Raghavan N, et al. Comparative metabolism of 14Clabeled apixaban in mice, rats, rabbits, dogs, and humans. Drug Metab Dispos. 2009;37:1738-1748.

21. Zhang D, He K, Herbst J, et al. Characterization of transporters involved in distribution and disposition of apixaban. Drug Metab Dispos. 2013;41:827-835

22. Yamahira N, Imai Y, Wastall P, et al. A placebo-controlled, ascending multiple-dose study to evaluate the safety, tolerability, pharmacokinetics, and pharmacodynamics of apixaban in healthy Japanese subjects (A637). Can J Clin Pharmacol. 2008;15:e719.

23. Yu Z, Nepal S, Bragat A, Shenker A, Frost C. Single dose apixaban pharmacokinetics and pharmacodynamics in healthy male Japanese and Caucasian subjects (A647). Can J Clin Pharmacol. 2008;15:e724.

24. Zhang D, He K, Raghavan N, et al. Metabolism, pharmacokinetics and pharmacodynamics of the factor Xa inhibitor apixaban in rabbits. J Thromb Thrombolysis. 2010;29:70-80.

25. Barrett YC, Wang J, Song Y, et al. A randomised assessment of the pharmacokinetic, pharmacodynamic and safety interaction between apixaban and enoxaparin in healthy subjects. Thromb Haemost. 2012;107:916-924.
Clinical Pharmacology: Advances and Applications

\section{Publish your work in this journal}

Clinical Pharmacology: Advances and Applications is an international, peer-reviewed, open access journal publishing original research, reports, reviews and commentaries on all areas of drug experience in humans. The manuscript management system is completely online and includes a very quick and fair peer-review system, which is all easy to use.

\section{Dovepress}

Visit http://www.dovepress.com/testimonials.php to read real quotes from published authors. 